\title{
Dispersive Raman spectroscopy for the in vitro identification and quantification of injected vancomycin intra-vitreous
}

\author{
Roney Carlos Lora ${ }^{\mathrm{a}}$, Landulfo Silveira Jr. ${ }^{\mathrm{b}, *}$, Stella Regina Zamuner ${ }^{\mathrm{c}}$ and \\ Marcos Tadeu Tavares Pacheco ${ }^{b}$ \\ ${ }^{a}$ Centro de Ciências da Saúde, Universidade Comunitária Regional de Chapecó, Chapecó, SC, Brazil \\ ${ }^{\mathrm{b}}$ Grupo de Engenharia Biomédica, Universidade Camilo Castelo Branco, Núcleo do Parque \\ Tecnológico de São José dos Campos, São José dos Campos, SP, Brazil \\ ${ }^{\mathrm{c}}$ Rehabilitation Sciences Master's Program, Nove de Julho University, São Paulo, SP, Brazil
}

\begin{abstract}
This work presents a study which aimed to quantify the intravitreally-injected vancomycin antibiotic using dispersive Raman spectroscopy and Partial Least Squares multivariate calibration. Eyes of New Zealand rabbits were injected with $5.0 \mathrm{mg}$ vancomycin at different time intervals $(8,24$ and $48 \mathrm{~h}$ groups). Distilled water was injected into the eyes of rabbits in the control group. The vitreous was then removed from each eyeball, placed into a quartz cuvette and submitted for dispersive Raman spectroscopy. The Raman equipment used a laser $(80 \mathrm{~mW}, 830 \mathrm{~nm})$, an imaging spectrograph and a CCD camera with exposure time of $50 \mathrm{~s}$. A calibration curve was developed using the PLS method with vancomycin diluted in water in the range of $1.0-8.0 \mathrm{mg} / \mathrm{ml}$. The spectrum of vancomycin was present in bands at the positions of $\mathrm{C}=\mathrm{C}$, amide $\mathrm{I}, \mathrm{CH}_{3}$, amide III and vibrations of the carbon chain $(\mathrm{C}-\mathrm{C}$, aromatic ring breathing and $\mathrm{C}-\mathrm{O}-\mathrm{C})$. The spectra of the injected vitreous bodies showed weak bands which were correlated with the pure drug. The PLS calibration model had a standard error of prediction of $0.8 \mathrm{mg} / \mathrm{ml}$ using one latent variable. The PLS showed changes in the predicted concentrations of the injected vitreous bodies depending on the time of injection, with an amount near zero for the water-injected group and ranging between $2 \mathrm{and} 3 \mathrm{mg} / \mathrm{ml}$ for the drug-injected groups. Raman spectroscopy could be employed in the quantitative evaluation of intravitreally-injected vancomycin in future in vivo applications.
\end{abstract}

Keywords: Raman spectroscopy, vancomycin quantification, diagnostic techniques, vitreous humour

\section{Introduction}

Ophthalmology is a medical specialty which benefits from non-invasive examinations for diagnostics and therapies mainly because of the delicate and extreme refinement of the eye structures and the noble sensory function performed by this organ [21]. Whenever a histological or cytological evaluation of the eyeball's interior is needed, a surgical intervention is performed. These interventions are aggressive and not commonly achieved via simple punctures, mainly because the eyes are filled, for the most part, by the vitreous gel, which consists of delicate and fragile filaments of type II collagen surrounded by water [3].

\footnotetext{
* Corresponding author: Landulfo Silveira Jr., Universidade Camilo Castelo Branco, Núcleo do Parque Tecnológico de São José dos Campos, Rod. Pres. Dutra, São José dos Campos, SP 12247-004, Brazil. Tel.: +55 123905 4401; E-mails: landulfo. silveira@unicastelo.br, landulfo.silveira@gmail.com.
} 
Nutrition and oxygenation of the vitreous body occur by diffusion from the vessels of the retina and ciliary body. The blood-ocular barrier provides an exchange of substances in an extremely selective way between the blood and the eye tissues [10]. This barrier is responsible for blocking the access of the great majority of medicines inside the eye and thus making it difficult for them to reach a minimal intraocular therapeutic concentration [10].

Endophthalmitis is an infectious disease that affects the interior of the eyes and is considered to be one of the most serious eye diseases, requiring a very rapid diagnosis and treatment $[8,17]$. The rapidness is essential, but there is a great clinical difficulty in identifying the infectious agent. In such cases, there is a need for surgical intervention to collect a biopsy sample for culturing to make an aetiological diagnosis [8]. In therapeutic terms, studies have shown that treatment with antibiotics is more effective when it is done via direct intraocular injections due to the difficulty the drugs have in crossing the ocular barrier systemically; this is the rule that has guided clinical conduct for endophthalmitis up until the present day [8].

In endophthalmitis, the infective microorganisms are generally gram-positive bacteria (between 60 and $80 \%$ ) or gram-negative bacteria (about 10-15\%). Vancomycin is the drug of first choice because it offers good bactericidal coverage for gram-positive microorganisms, and when combined with amikacin, which provides good coverage for gram-negative microorganisms, satisfactory results can be achieved $[8,17]$. The dose commonly used in the vitreous is $1 \mathrm{mg}$ dissolved in a volume of $0.1 \mathrm{ml}(10 \mathrm{mg} / \mathrm{ml})$ for direct injections into the vitreous chamber. These parameters have been shown to be effective in controlling the infection with a low toxicity to the retina [8].

The problem faced out when using direct intraocular applications is the maintenance of a minimum concentration that causes the bactericidal effect [17]. The usual clinical conduct is to reapply another dose of vancomycin after $48 \mathrm{~h}$ if clinical examination still shows evidence of infection [26]. The difficulties faced by clinicians are the evaluation of the concentration level after reapplication, when metabolism and interactions with the inflammatory process take place, and the avoidance of excessive concentrations which could lead to retinal toxicity [6].

In clinical practice, the ability to identify structures and quantify substances inside the eye without the need for surgical collection is urgently required. Optical techniques such as fluorescence [2] and near-infrared Raman spectroscopy [23] have been considered as important tools for helping to identify changes in the biological structures of the eye due to the ease of access to the eye's morphological structures and its ability to transmit and scatter visible and near-infrared light [1].

The potential of Raman spectroscopy being used as a diagnostic tool in different tissues and body fluids has been studied $[5,12,13,15,25]$. The diagnosis of human tissue pathologies could be addressed due to the high chemical and structural information content of this spectroscopic technique [18]. A Raman spectrum shows well-resolved and narrow bands originating from the inelastic scattering of the incident photons by the molecular vibrational modes of the chemical bonds of biomolecules [13]. The spectral information revealed by Raman spectroscopy can be used for qualitative and quantitative analyses of samples $[13,19]$. Due to the high transparency of internal tissues of the eye to infrared light, one could stimulate and capture the Raman spectrum from molecules inside the eye [11].

The use of Raman spectroscopy to evaluate eye structures and contents has been demonstrated with rapid data acquisition and minimal invasion; for example, the evaluation of intraocular metabolites such as glucose, lactate and urea [27], the crystalline molecular changes in cataracts [20], the detection of macular carotenoid levels in the retina [4] and non-enzymatic glycation in the vitreous of patients with diabetic retinopathy [22]. In the vitreous gel in particular, being a tissue composed almost entirely of water with a low concentration of collagen fibres, Raman spectroscopy presents a real possibility for 
identifying composition changes as the normal vitreous does not present significant Raman signals in the fingerprint region $\left(600-2000 \mathrm{~cm}^{-1}\right)$. Drugs could be quantified in the eye's anterior chamber at concentrations closer to the minimum inhibitory concentration [23].

Chemometrics is a mathematical and statistical tool for quantitatively analysing the chemical data from multiple sources which arose from the need to treat and/or to obtain information from multichannel equipment, such as spectrographs, for example, where the intensity of the absorption, scattering or emission for a range of wavelengths are recorded in only one spectrum at a time [7]. One of the chemometric tools of great importance in the analytical chemistry field is partial least squares (PLS). It is an efficient method for dealing with experimental noise, colinearities and nonlinearities, being sufficiently robust to permit the development of a calibration model in which the parameters do not change considerably with the inclusion of new samples to the data set, even in the presence of interferences, whether known or unknown [7,9].

The PLS method can be divided into two steps. The first consists of mounting the $X$ matrix from the spectral data (independent variables) and the $Y$ matrix from the properties of interest, or the concentrations, for example (dependent variables). The second step consists of obtaining the coefficients showing the relationship between the $X$ and $Y$ matrices, which demonstrates the property of interest, i.e., the correlation between the concentration and the spectral variation. Then the results can be used to estimate the concentrations of new samples based on the spectra of the original samples [9]. In this way, PLS effectively measures the concentrations of a solute in solution, and is useful in helping to determine the concentration of the active ingredient of a drug in a biological fluid or tissue via Raman spectroscopy.

The objective of this study was to evaluate the viability of a near-infrared dispersive Raman spectroscopy technique as a tool to: (a) identify vancomycin molecules injected intravitreally $(5.0 \mathrm{mg})$ into New Zealand rabbits at different time intervals $(8,24$ and $48 \mathrm{~h}$ ), (b) to quantify the injected vancomycin by developing a calibration model using multivariate PLS (partial least squares) applied to the Raman spectra of diluted vancomycin in distilled water $(1.0-8.0 \mathrm{mg} / \mathrm{ml})$ and (c) to use such a model to estimate the drug concentration in the Raman spectra of injected vitreous samples in vitro.

\section{Materials and methods}

This study was approved by the Committee of Ethics in Research of Universidade do Vale do Paraíba. Twelve New Zealand rabbits were used, each weighing approximately $800 \mathrm{~g}$, divided into three groups of four animals per group. These animals were chosen due to the morphological and structural similarities of their eyes to human eyeballs and the ease of access and manipulation. The groups correspond to the vancomycin injection times of $8 \mathrm{~h}(\mathrm{G} 1), 24 \mathrm{~h}(\mathrm{G} 2)$ and $48 \mathrm{~h}(\mathrm{G} 3)$. Vancomycin was injected into a rabbit's vitreous humour after the anaesthesia protocol, which consisted of an intramuscular injection of $0.3 \mathrm{ml}$ of Zoletil 50 (tiletamine chlorhydrate $125 \mathrm{mg}$, zolazepam chlorhydrate $125 \mathrm{mg}$ ), and the animal handling was done after about $15 \mathrm{~min}$ of anaesthesia. Then, a single dose of $5 \mathrm{mg}$ of vancomycin diluted in $0.05 \mathrm{ml}$ distilled water was injected into the vitreous gel of both eyes of each rabbit. One animal of each group was injected with $0.05 \mathrm{ml}$ distilled water in the vitreous of both eyes as the control. In order to sacrifice all of the animals on the same day for Raman collection the injections followed a sequence whereby the G3 group were injected on the first day, the G2 group on the second day and the G1 group on the last day before euthanasia.

During the experiment, all animals were fed with proper food and ad libidum water and showed no significant changes in behaviour and no clinically observable changes in their eyes. Following the appropriate period after injection, all of the animals were sacrificed and both eyeballs removed. 
For the euthanasia, a deep anaesthesia with $0.5 \mathrm{ml} \mathrm{Zoletil}{ }^{\circledR}$ was followed by an overdose of intracardiac potassium chloride. Immediately after sacrifice, the eyeballs were removed via micro-surgery and the vitreous content was separated from the other structures in the eye and collected. The approximate volume of each vitreous ranged from 0.8 to $1 \mathrm{ml}$. The vitreous content of each eyeball from each animal was placed in a quartz cuvette and analyzed using dispersive Raman spectroscopy on the same day as sacrifice.

The Raman spectrum was obtained by a laboratory dispersive near-infrared Raman spectrometer, as described elsewhere [24]. Briefly, it uses a diode laser with an $830 \mathrm{~nm}$ excitation wavelength and an $80 \mathrm{~mW}$ output power directed towards a sample holder with the cuvette for sample irradiation. This sample holder keeps a $45^{\circ}$ angle scattering geometry with the incident light. The backscattered light is collected by lenses placed at the spectrograph's entrance. The scattered excitation light is blocked at the spectrograph's entrance by a laser-rejection notch filter. The light is then dispersed by an imaging spectrograph with a spectral resolution of about $10 \mathrm{~cm}^{-1}$, with a slit of about $150 \mathrm{~cm}^{-1}$, which was found to have an optimal signal-to-noise ratio and spectral resolution [16]. This spectrograph is coupled to a CCD camera (cooled by liquid nitrogen, deep depletion) and a camera controller is connected to a computer for the detection of collected light. A software program controls detector exposure time and the number of acquisitions per sample. The exposure time for obtaining the Raman spectra was selected so that there was no saturation of the CCD and was set to $50 \mathrm{~s}$. The spectra were collected in duplicate.

After acquisition, the spectra were pre-processed by fitting and subtracting the background (mainly fluorescence) with a fifth order polynomial in the spectral range between 800 and $1800 \mathrm{~cm}^{-1}$, and cosmic ray spikes were manually removed. The spectrum of water $(0 \mathrm{mg} / \mathrm{ml}$ drug concentration) was collected and subtracted from all spectra to remove the Raman contribution from the cuvette and any CCD readout pattern such as cold or hot pixels. The spectrum of lyophilized vancomycin was also collected.

In order to quantify the concentration of intravitreally-injected vancomycin presented in the samples of rabbit's eyeballs, a calibration curve was developed using the spectra of vancomycin diluted in distilled water. Duplicate Raman spectra of vancomycin dilutions in the concentrations of 1.0, 2.0, 3.0, 4.0, 6.0 and $8.0 \mathrm{mg} / \mathrm{ml}$ in a quartz cuvette were obtained. Using the known concentrations of vancomycin in water and the intensity of the Raman spectra, a multivariate calibration model was developed which applied PLS to a set of spectra which were used to calculate drug concentrations in the vitreous based on the Raman spectra of such samples. In Matlab (version 7.0), the Raman spectra and the concentrations of vancomycin were considered as the independent (ordinate) and dependent (abscissa) variables, respectively. Then the PLS model was built using the number of latent variables set to one, since there was just one important variable, the injected drug, to be considered in the spectra.

In order to test the prediction ability of the model, a cross-validation procedure was established using the replicated spectra of diluted vancomycin with the latent variable also set to one. Then a scatter plot was made of the diluted versus predicted concentrations and the model's standard error of prediction (SEP) was calculated. The SEP evaluates the accuracy of the PLS model in predicting concentrations within the range used in the model [9] and it also verifies that such errors are within acceptable values in the literature and in clinical practice. It was calculated as follows [7,9]:

$$
\operatorname{SEP}(\%)=\sqrt{\frac{\sum_{i=1}^{n}\left(C_{i}-C a_{i}\right)^{2}}{\sum_{i=1}^{n} C a_{i}^{2}}} \times 100,
$$

where $C a_{i}$ is the real concentration of the sample and $C_{i}$ is the concentration predicted by the PLS model. 


\section{Results}

Figure 1 presents the Raman spectrum of a sample of lyophilized vancomycin used in the vitreous injection. The spectrum shows distinctive Raman bands, related to the vibrational modes of vancomycin molecules. It shows a tentative attribution for the most important bands based on the literature of organic Raman bands [14,25]. The band at $1696 \mathrm{~cm}^{-1}$ can be attributed to $\mathrm{C}=\mathrm{C}$ stretching, the band at $1625 \mathrm{~cm}^{-1}$ to the carbonyl group $\left(\mathrm{C}=\mathrm{O}\right.$ stretching) of amide $\mathrm{I}$, the band at $1475 \mathrm{~cm}^{-1}$ can be attributed to $\mathrm{CH}_{3}$ bending, the bands around $1327 \mathrm{~cm}^{-1}$ can be attributed to $\mathrm{CH}_{2}$ and $\mathrm{CH}_{3}$ deformation: twisting and wagging modes, the band at $1252 \mathrm{~cm}^{-1}$ can be attributed to amide III (C-N stretching), and the bands in the $1200-800 \mathrm{~cm}^{-1}$ range can be attributed to the stretching vibrations of the carbon chain. The band at $1145 \mathrm{~cm}^{-1}$ can be attributed to $\mathrm{C}-\mathrm{C}$ stretching, the band at $1004 \mathrm{~cm}^{-1}$ represents breathing of the aromatic ring, the band at $900 \mathrm{~cm}^{-1}$ represents the stretching of the $\mathrm{C}-\mathrm{C}$ bond and, finally, the band at $842 \mathrm{~cm}^{-1}$ represents the deformation of $\mathrm{C}-\mathrm{O}-\mathrm{C}$.

In order to develop a model for calculating the concentrations of intravitreally-injected vancomycin through Raman spectroscopy, a serial dilution of vancomycin in distilled water was performed in duplicate and submitted to the spectrometer. Figure 2 shows the Raman spectra of different concentrations of vancomycin in aqueous solution, taken in a quartz cuvette, at concentrations of 1.0, 2.0, 3.0, 4.0, 6.0 and $8.0 \mathrm{mg} / \mathrm{ml}$. The spectrum of $0 \mathrm{mg} / \mathrm{ml}$ was considered background and subtracted from all spectra of diluted vancomycin. The spectra were not smoothened so the original signal-to-noise ratio characteristic was maintained since this did not affect the PLS calibration and smoothing could introduce artifacts such as Raman bands in positions where they are not expected.

The spectra of diluted vancomycin were used to develop a calibration model based on PLS, which employed all of the available spectral information within the range of $1.0-8.0 \mathrm{mg} / \mathrm{ml}$ concentrations, with the aim of quantifying the concentration of vancomycin injected into the vitreous. The multivariate calibration provides a significant improvement in the prediction capability when compared to a single peak/band analysis because it takes into account not only the intensity of the bands, but also the widths and positions, relating all of the spectral information that is correlated with the sample variations of intensity. Figure 3 shows the spectra of the latent variables 1 and 2. It can be observed that LV1 is closely

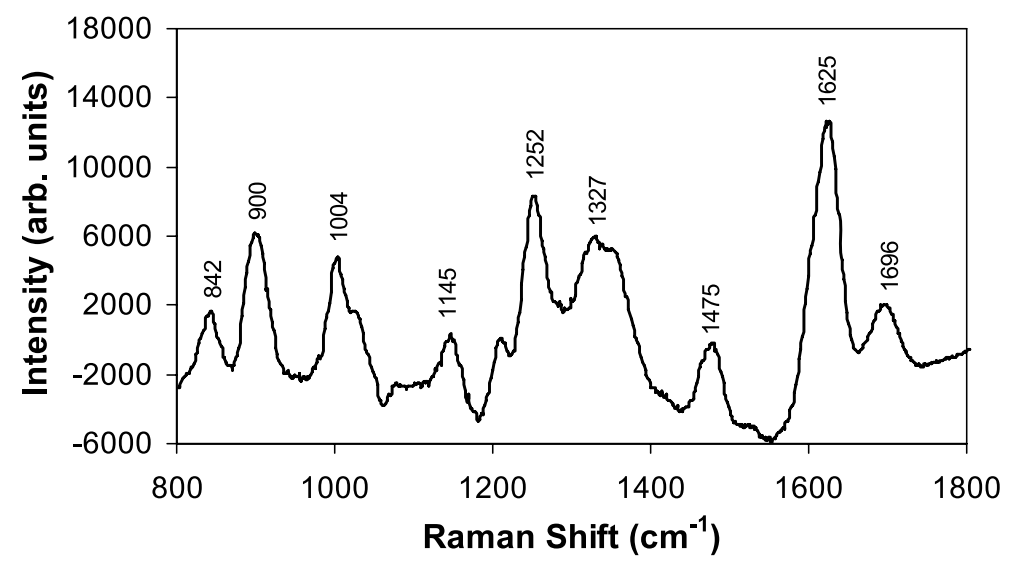

Fig. 1. Raman spectrum of lyophilized vancomycin taken with the drug placed in a quartz cuvette. Laser power: $80 \mathrm{~mW}$, laser wavelength: $830 \mathrm{~nm}$, resolution: $10 \mathrm{~cm}^{-1}$. 


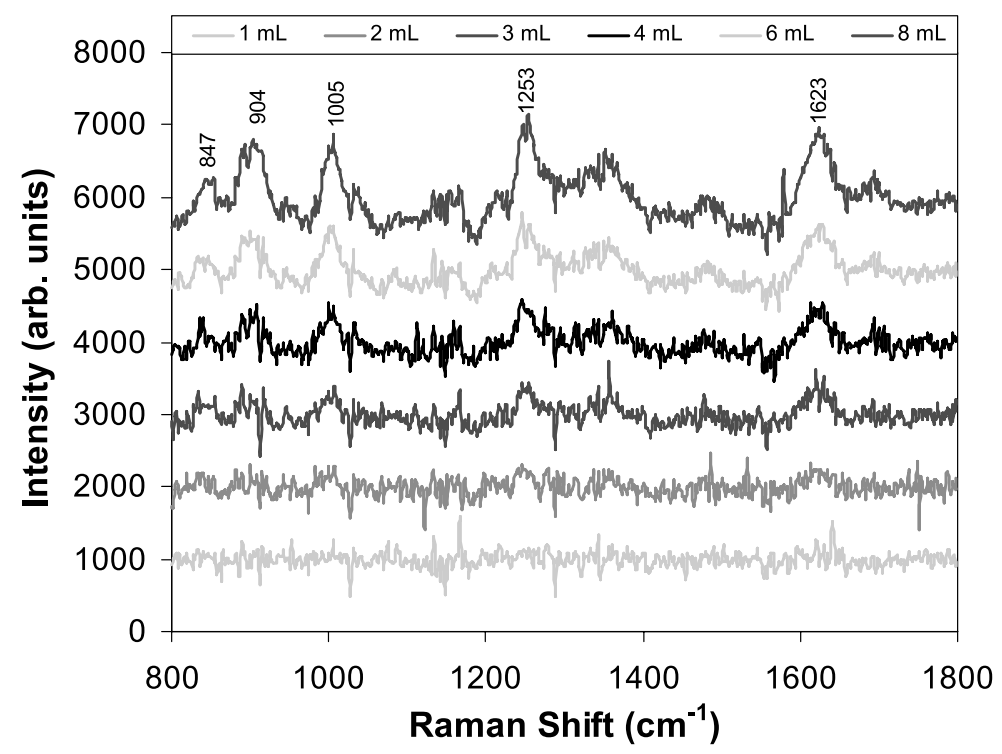

Fig. 2. Raman spectra of vancomycin diluted in distilled water with known concentrations $(\mathrm{mg} / \mathrm{ml})$ after subtracting the water spectrum. Spectra were plotted with an offset in the intensity axis to evidence the differences in relative intensity of Raman bands for different concentrations. Laser power: $80 \mathrm{~mW}$, laser wavelength: $830 \mathrm{~nm}$, resolution: $10 \mathrm{~cm}^{-1}$.
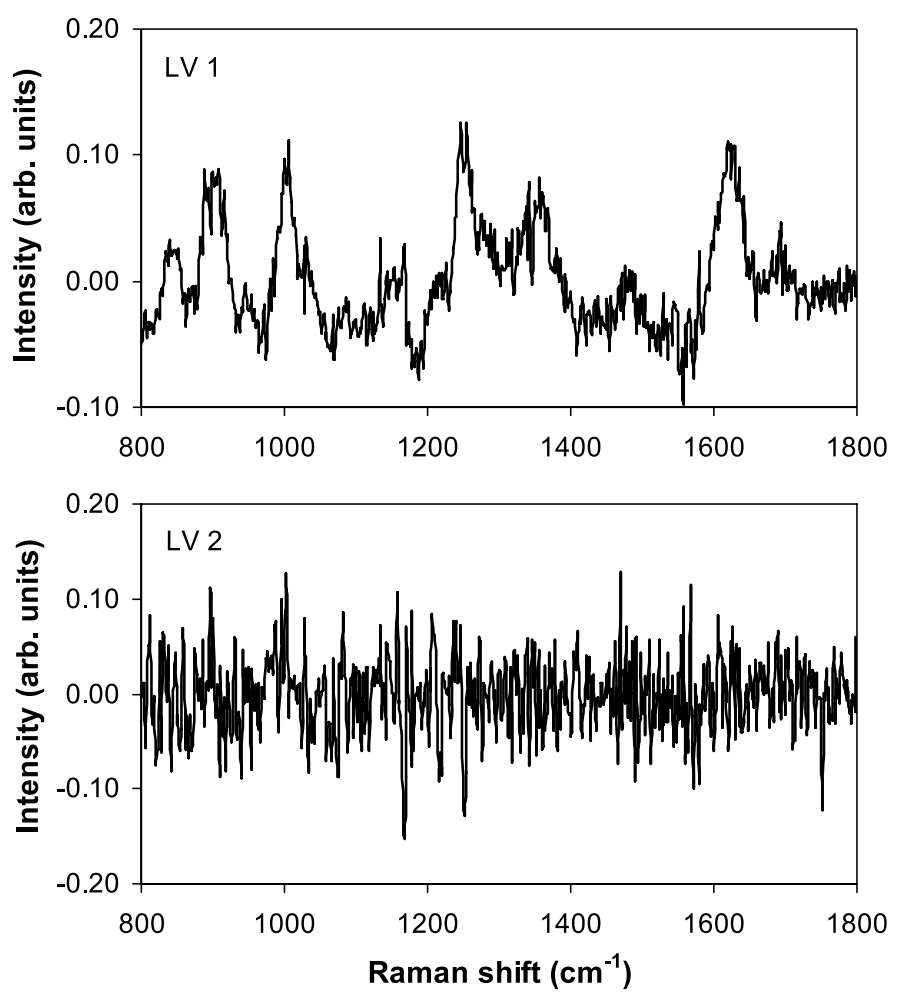

Fig. 3. Plot of the first two latent variables (LV1 and LV2) of the PLS model using the spectra of vancomycin diluted in water. 


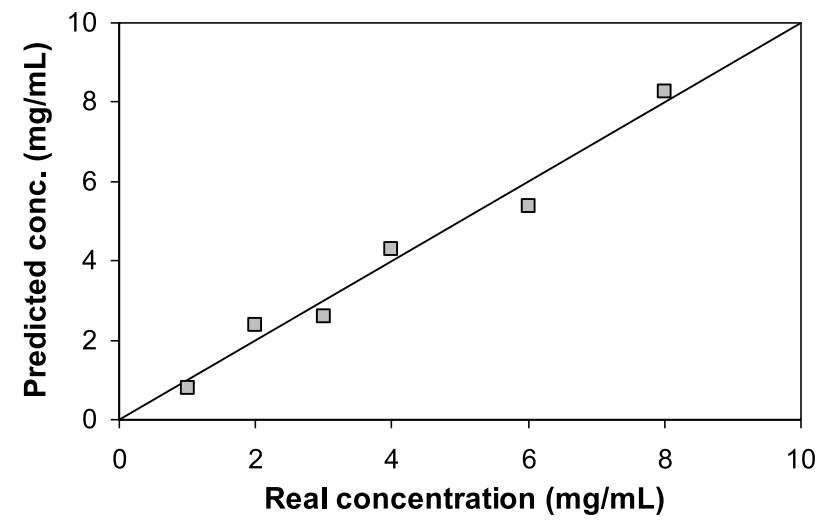

Fig. 4. Plot of the predicted concentration versus the real concentration of vancomycin diluted in water calculated by the PLS model applied to the Raman spectra of replicate samples using one latent variable (LV1). SEP $=0.8 \mathrm{mg} / \mathrm{ml}$.

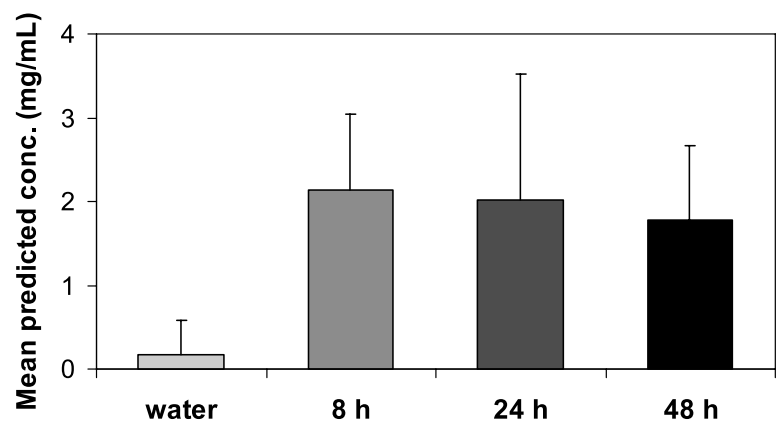

Fig. 5. Plot of the mean and standard deviation values of drug concentration predicted by PLS for each injection group (control water; G1 $-8 \mathrm{~h} ; \mathrm{G} 2-24 \mathrm{~h} ; \mathrm{G} 3-48 \mathrm{~h}$ ). ANOVA indicated that statistically significant difference was achieved between water and all injected groups $(p=0.01)$.

related to the spectrum of vancomycin, while LV2 does not provide relevant spectral information. Therefore, in this work, the PLS model only used LV1 because there was only one set of spectral information, the vancomycin, sufficient for explaining the spectral variations found in the data set. Figure 4 shows the result of the cross-validation applied to the replicate dilutions of vancomycin in water, where the concentrations predicted by the PLS were plotted versus the real concentrations. The SEP for the PLS model was calculated as $0.8 \mathrm{mg} / \mathrm{ml}$.

In order to evaluate the intravitreally-injected vancomycin $(8,24$ and $48 \mathrm{~h})$ in the experimental groups, the Raman spectra of the vitreous of each eye from each animal were collected (six spectra - two vitreous bodies from of three animals per group) and submitted to the PLS model with the parameters defined in the calibration. Then the predicted concentration for each injected group was averaged and plotted in Fig. 5. It was found that the amount of intravitreous vancomycin was very low in the distilled waterinjected controls, indicating that no drug had been found. This value in the eyes changed depending on the time of injection, being higher for the $8 \mathrm{~h}$ and lower for the 24 and $48 \mathrm{~h}$ injections. An ANOVA test with 5\% significance indicated that there was no statistically significant difference between the druginjected groups, but a statistically significant difference was found between all drug-injected groups and the water-injected group $(p=0.01)$. 


\section{Discussion}

The study focused on the vitreous body in the rabbit, consisting of water (99\%), type II collagen and hyaluronic acid (1\%). It can be seen in Fig. 4, which shows the spectrum of vitreous without the injection of any drug, that no significant Raman bands were observed in this group. This was also shown in the study by Sebag et al. [22], who found a very weak Raman signal of pure human vitreous, even in the dehydrated form. In the spectra of vitreous injected with vancomycin at different time intervals, one can observe that the inoculated groups showed similar bands, with an emphasis on the bands close to 904, 1005 and $1623 \mathrm{~cm}^{-1}$ (Fig. 4).

An increase in the mean predicted concentration was found for all groups with the injected drug, as well as a gradual reduction over time $(8>24>48 \mathrm{~h})$. The decrease over time can be explained by the metabolism of the drug inside the vitreous chamber [17]. The high standard deviation for the samples with the drug predicted by the PLS (from 0.8 to $1.5 \mathrm{mg} / \mathrm{ml}$ ) was corroborated by the model's high SEP value $(0.8 \mathrm{mg} / \mathrm{ml})$. However, this fact did not impede the employment of the PLS model in the calculation of drug concentrations or the demonstration of differences depending upon the time of injection as presented inside the vitreous.

This high SEP could be attributed to the low signal-to-noise ratio for the samples used in the calibration, in which the Raman signal would have been covered by the electronic readout and the dark current noise, also contributing to the larger error bars in the data collected from vitreous. Also, contamination of the vitreous by other eye structures during dissection of the vitreous could worsen the signal and introduce artifacts that could be interpreted as bands in the PLS model. However, it is also possible that non-regular diffusion of the drug inside the vitreous could lead to a laser irradiation and Raman collection in a vitreous site with an insufficient concentration of vancomycin. The dose of vancomycin was administered into the vitreous body in one single injection, and from this site the drug should have diffused throughout the rest of the vitreous body.

In order to increase the reliability of the predicted concentrations by the PLS, one could homogenize the vitreous. Also, an increase in laser power would improve the Raman signal-to-noise ratio of the vitreous. One could also use a laser with a shorter wavelength, since the scattering increases inversely with the wavelength. However, one inconvenience of this method would be that a smaller wavelength would have a more intense fluorescence background. Some authors have shown that a smaller wavelength excitation (argon laser, $514.5 \mathrm{~nm}$ ) can be used to increase the signal-to-noise ratio of Raman spectra, as shown by Sideroudi et al. [23], but with a disadvantage of the lower penetration depth in ocular tissues with an intense opacity caused by endophthalmitis. The use of a micro-Raman system would enable the evaluation of even small fragments of biological tissues selectively [11]. Furthermore, the calibration curve could be developed using more closely spaced dilutions in the range of $0.5-5.0 \mathrm{mg} / \mathrm{ml}$, closer to the concentrations found in vivo.

In terms of diagnosis, the use of Raman spectroscopy would represent great progress since it permits a rapid and non-invasive procedure for assessing the molecular composition of the vitreous chamber. In other words, in the presence of endophthalmitis, the optical diagnosis performed via Raman spectra could indicate the possible need for therapy supplementation.

\section{Conclusion}

This work demonstrated that dispersive Raman spectroscopy could detect injected vancomycin in the vitreous chamber of rabbits in vitro using a calibration curve based on partial least squares (PLS) 
applied to Raman spectra of vancomycin diluted in water, with a prediction error of about $0.8 \mathrm{mg} / \mathrm{ml}$. Although there were larger error bars for the concentrations predicted in vitreous tissues, this technique is a promising application and could be exploited as a tool for identifying vancomycin injected inside the eyes.

\section{Acknowledgement}

L. Silveira Jr. thanks CNPq (Conselho Nacional de Desenvolvimento Científico e Tecnológico) for the Productivity Award fellowship (PQ2-305610/2008-2).

\section{References}

[1] P.V. Algvere, P.L. Torstensson and B.M. Tengroth, Light transmittance of ocular media in living rabbit eyes, Investigative Ophthalmology and Visual Science 34 (1993), 349-354.

[2] A. Ayata, M. Unal, D. Ersanli and S. Tatlipinar, Near infrared fluorescence and OCT features of choroidal abnormalities in type 1 neurofibromatosis, Clinical and Experimental Ophthalmology 36 (2008), 390-392.

[3] E.A. Balazs, The vitreous, International Ophthalmology Clinics 13 (1973), 169-187.

[4] P.S. Bernstein, M.D. Yoshiba, N.B. Katz, R.W. McClane and W. Gellermann, Raman detection of macular carotenoid pigments in intact human retina, Investigative Ophthalmology and Visual Science 39 (1998), 2003-2011.

[5] B. Bodanese, L. Silveira, R. Albertini, R.A. Zângaro and M.T.T. Pacheco, Differentiating normal and basal cell carcinoma human skin tissues in vitro using dispersive Raman spectroscopy: a comparison between Principal Components Analysis and simplified biochemical models, Photomedicine and Laser Surgery 28(1) (2010), S119-S127.

[6] P.A. Campochiaro and W.R. Green, Toxicity of intravitreous ceftazidime in primate retina, Archives of Ophthalmology 110 (1992), 1625-1629.

[7] A.P. Cunha, V.P.P. Cunha, L. Silveira and A.A. Martin, Calibration model of methylmetacrylate concentration in aqueous solution using ultraviolet absorption spectroscopy, Quimica Nova 26 (2003), 850-854.

[8] M.J. Elder and N. Morlet, Endophthalmitis, Clinical and Experimental Ophthalmology 30 (2002), 394-398.

[9] M.M.C. Ferreira, A.M. Antunes, M.S. Melgo and P.L.O. Volpe, Chemometrics I: multivariate calibration, a tutorial, Quimica Nova 22 (1999), 724-731.

[10] W.S. Foulds, Basic aspects and practical considerations in retinal detachment surgery, Clinical and Experimental Ophthalmology 14 (1986), 113-120.

[11] J.V. Glenn, J.R. Beattie, L. Barrett, N. Frizzell, S.R. Thorpe, M.E. Boulton, J.J. McGarvey and A.W. Stitt, Confocal Raman microscopy can quantify advanced glycation end product (AGE) modifications in Bruch's membrane leading to accurate, nondestructive prediction of ocular aging, FASEB Journal 21 (2007), 3542-5352.

[12] A.S. Haka, K.E. Shaper-Peltier, M. Fitzmaurice, J. Crowe, R.R. Dasari and M.S. Feld, Diagnosing breast cancer by using Raman spectroscopy, Proceedings of the National Academy of Science 102 (2005), 12371-12376.

[13] E.B. Hanlon, R. Manoharan, T.W. Koo, K.E. Shafer, J.T. Motz, M. Fitzmaurice, J.R. Kramer, I. Itzkan, R.R. Dasari and M.S. Feld, Prospects for in vivo Raman spectroscopy, Physics in Medicine and Biology 45 (2000), R1-R59.

[14] Horiba Jobin Yvon, Raman data and analysis, Raman application note, 2008, available at: http://www.horiba.com/ fileadmin/uploads/Scientific/Documents/Raman/bands.pdf.

[15] C. Krafft and V. Sergo, Biomedical applications of Raman and infrared spectroscopy to diagnose tissues, Spectroscopy International Journal 20 (2006), 195-218.

[16] J.C. Lázaro, M.T.T. Pacheco, K.C. Rodrigues, C.J. Lima, L.M. Moreira, A.B. Villaverde and L. Silveira, Optimizing the Raman signal for characterizing organic samples: The effect of slit aperture and exposure time, Spectroscopy International Journal 23 (2009), 71-80.

[17] C.A. Lemley and D.P. Han, Endophthalmitis: a review of current evaluation and management, Retina 27 (2007), 662-680.

[18] R. Manoharan, Y. Wang and M.S. Feld, Histochemical analysis of biological tissues using Raman spectroscopy, Spectrochimica Acta Part A $\mathbf{5 2}$ (1996), 215-249.

[19] L.M. Moreira, L. Silveira, F.V. Santos, R. Rocha, R.A. Zângaro, A.B. Villaverde and M.T.T. Pacheco, Raman spectroscopy: a powerful technique for biochemical analysis and diagnosis, Spectroscopy International Journal 22 (2008), $1-19$.

[20] S. Nie, K.L. Bergbauer, J.F.R. Kuck and N.T. Yu, Near infrared Fourier transform Raman spectroscopy in human lens research, Experimental Eye Research 51 (1990), 619-623. 
[21] K. Pesudovs and D.J. Coster, Assessment of visual function in cataract patients with a mean visual acuity of 6/9, Australian and New Zealand Journal of Ophthalmology 24 (1996), 5-9.

[22] J. Sebag, S. Nie, K. Reiser, M.A. Charles and N.T. Yu, Raman spectroscopy of human vitreous in proliferative diabetic retinopathy, Investigative Ophthalmology and Visual Science 35 (1994), 2976-2980.

[23] T.I. Sideroudi, N.M. Pharmakakis, G.N. Papatheodorou and G.A. Voyiatzis, Non-invasive detection of antibiotics and physiological substances in the aqueous humor by Raman spectroscopy, Lasers in Surgery and Medicine 38 (2006), 395403.

[24] L. Silveira, L.M. Moreira, V.G.B. Conceição, H.L. Casalechi, I.S. Muñoz, F.F. Silva, M.A.S.R. Silva, R.A. Souza and M.T.T. Pacheco, Determination of sucrose concentration in lemon-type soft drinks by dispersive Raman spectroscopy, Spectroscopy International Journal 23 (2009), 217-226.

[25] N. Stone, C. Kendall, J. Smith, P. Crow and H. Barr, Raman spectroscopy for identification of epithelial cancers, Faraday Discussions 126 (2004), 141-157.

[26] W. Tasman, The Wills Eye Hospital: Atlas of Clinical Ophthalmology, Lippincott Williams \& Wilkins, Philadelphia, PA, 2001.

[27] J.P. Wicksted, R.J. Erckens, M. Motamedi and W.F. March, Raman spectroscopy studies of metabolic concentrations in aqueous solutions and aqueous humor specimens, Applied Spectroscopy 49 (1995), 987-993. 


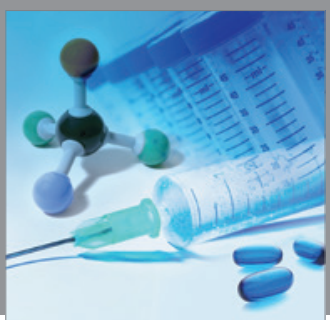

International Journal of

Medicinal Chemistry

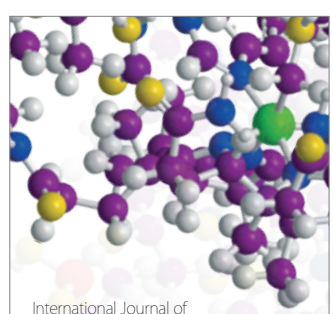

Carbohydrate Chemistry

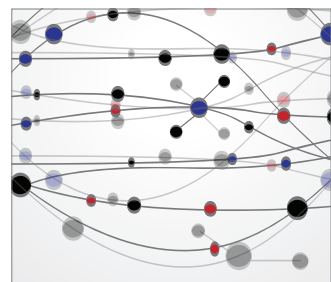

The Scientific World Journal
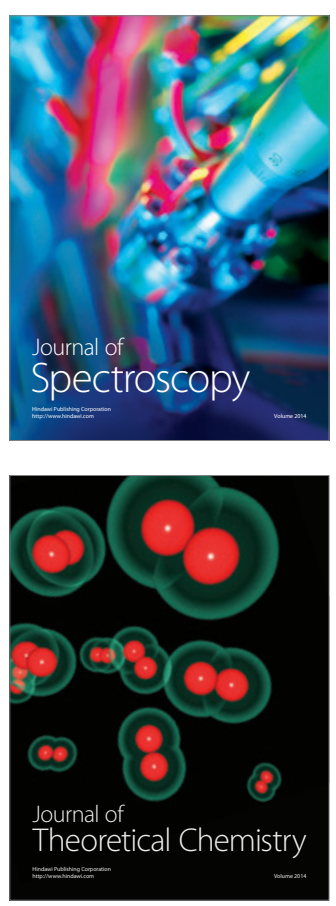
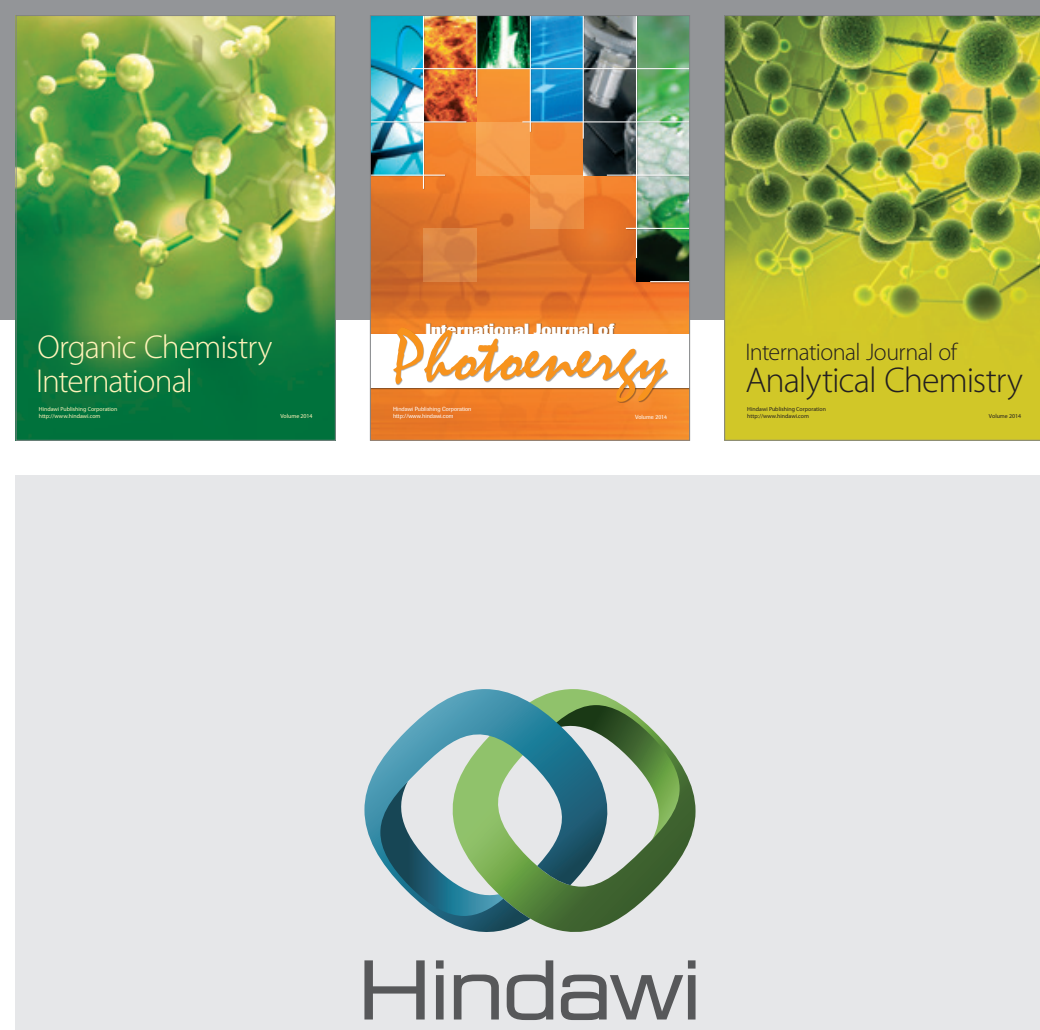

Submit your manuscripts at

http://www.hindawi.com
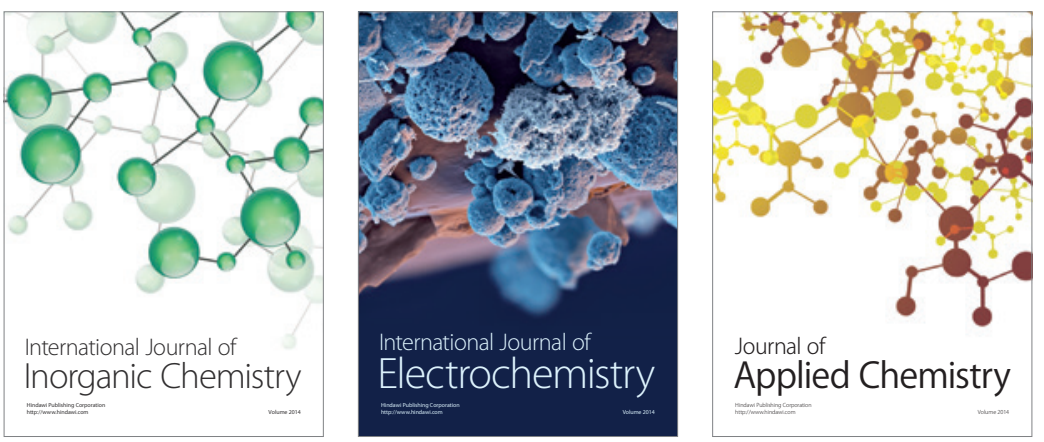

Journal of

Applied Chemistry
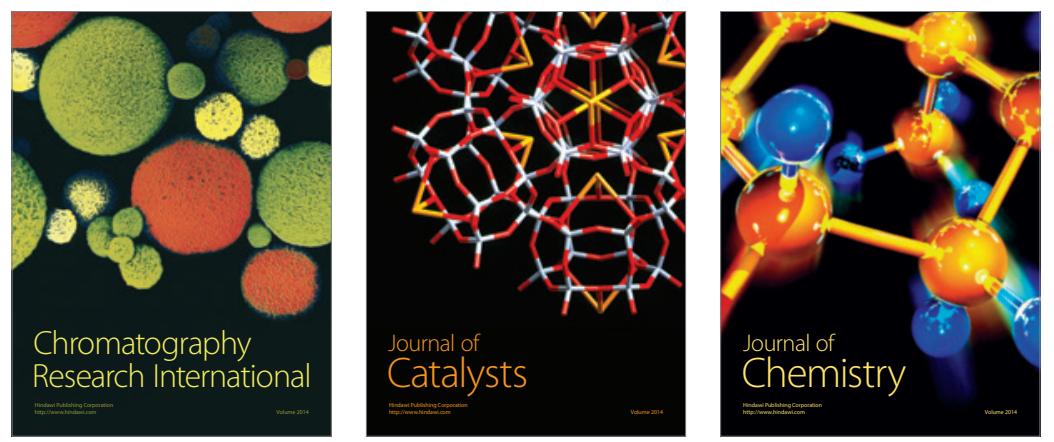
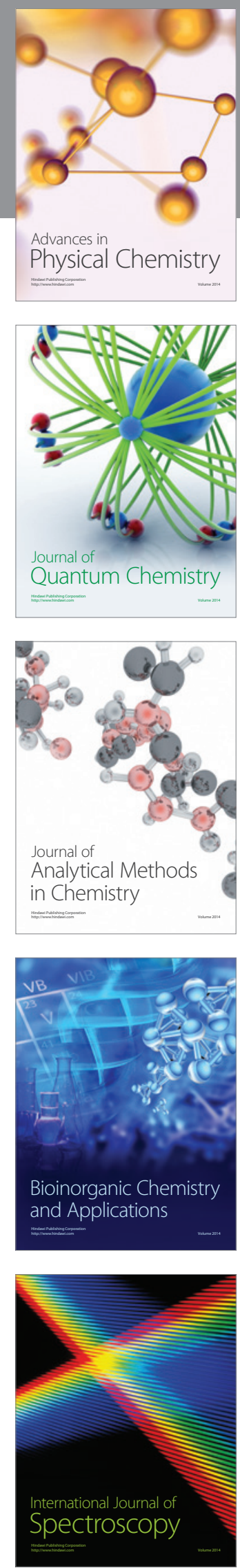